\title{
Fractionation of Poly(ethylene oxide) Star Samples by Supercritical Fluids
}

\author{
François Cansell, Philippe Botella, Jean-Luc Six, ${ }^{*}$ Yves Garrabos, \\ Roland Tufeu, ${ }^{* *}$ and Yves GNANOU* \\ Institut de Chimie de la Matière Condensée de Bordeaux, CNRS-Université Bordeaux I, \\ Château Brivazac, 33608 Pessac Cedex-France \\ * Laboratoire de Chimie des Polyméres Organiques, ENSCPB-Université Bordeaux I-CNRS, \\ 351, cours de la Libèration-33405 Talence Cedex-France \\ ** Laboratoire d'Ingénierie des Matériaux et des Hautes Pressions, CNRS-Université Paris Nord, \\ Avenue J.B. Clement, 93430 Villetaneuse-France
}

(Received March 21, 1997)

\begin{abstract}
Supercritical fluids have been used for the fractionation of poly(ethylene oxide). Indeed, supercritical fluids are well known for their efficiency in the fractionation of polymers. Polymer fractions of similar or lower molar mass distribution can be isolated with higher purity and lower energy costs than with other fractionation methods. The fractionation of poly(ethylene oxide) (POE) is reported at $423 \mathrm{~K}$ in the pressure range $8--35 \mathrm{MPa}$. Used as supercritical fluid $\mathrm{HCFC} 142 \mathrm{~b}$ (1-chloro-1,1-difluoroethane, $\mathrm{CH}_{3} \mathrm{CClF}_{2}$ ) was found to be quite efficient, yielding polymers with a very narrow molar distribution. These results enable tochose the optimum pressure conditions for molecular architecture fractionation. The fractionation of a binary mixture of star and linear chains of poly(ethylene oxide) has been studied. The separation of linear chains and star structure appears to be very efficient for a short elution time.
\end{abstract}

KEY WORDS Star Structure / Fractionation / Supercritical Fluid / Poly(ethylene oxide) /

Star polymers have been the subject of keen interest over the last decade because they were found to exhibit quite peculiar behavior both in solution and in bulk. Most of the start polymers have been so far prepared via the so-called "arm-first" method. ${ }^{1}$ This strategy consists in deactivating a "living" linear polymer on a plurifunctional core or alternatively in copolymerizing "living" chains with a difunctional monomer. To obtain start samples with exactly the same functionality as that of the plurifunctional deactivating agent, it is requested to use a rather large excess of living precursor chains. A fractionation step is therefore required to separate the star molecules from linear contaminants. This is generally effected by fractional precipitation upon incrementally adding a non-solvent to the polymer solution; but this procedure is time consuming and tedious. Such drawbacks (use of an excess of linear chain and necessity for fractionation) can theoretically be avoided whenever stars are obtained by the so-called "core-first" method, which involves the use of a plurifunctional initiator. Provided the initiation reaction is quantitative and the functionality of the initiator precisely defined, stars of predetermined size and functionality are directly accessible. $^{2}$

Even though the synthesis of poly(ethylene oxide) (PEO) stars from a plurifunctional alcoholate initiator meets the above requirements, ${ }^{3}$ the PEO star samples are often contaminated with linear materials. These linear chains are induced by monofunctional alcoholates that result from the deprotonation of residual protonic impurities present in the reaction medium at the time of the initiation. Because fractional precipitation by a non-solvent does not satisfactorily work below the crystalline melting temperature of PEO $\left(66^{\circ} \mathrm{C}\right)$, size exclusion chromatography and dialysis can represent alternative approches. Unfortunately, these techniques are not well suited to obtain large quantities of separated products.

In order to improve and optimize the efficiency of the separation, in time and in mass, we came to consider the use of supercritical fluids (SCF). Indeed, the SCF have specific properties: in particularly they are characterized by a low viscosity and a large density. By small changes in pressure or temperature conditions, it is possible to strongly modify the density, and the solubility of the solute. Many studies have been recently devoted to processes using SCF ${ }^{6}$ An important application of SCF is the fractionation of a broadly distributed polymer into narrow fractions. ${ }^{7-16}$ In the fractionation of a given polymer, the main parameter is the separation factor which is the ratio between the equilibrium concentraions of a $i$-mer (a polymer with a $i$ degree of polymerization) in the coexisting two phases. ${ }^{17}$ The mass-based partition coefficient can be described by the following equation ${ }^{18}$ :

$$
\log \left(w_{i}^{\prime} / w_{i}\right)=\log K_{i}=-\sigma M_{i}+\beta
$$

The $K_{i}$ factor, which represents the ratio of the mass fraction of the $i$-mer in the solvent rich phase $\left(w_{i}^{\prime}\right)$ and so its mass fraction in the polymer rich phase $\left(w_{i}\right)$, is a function of both temprature and pressure at which the fraction is carried out. The $i$-mer solubility is controlled by the medium density and increases with this latter one. At constant pressure and temperature, the variation of this $K_{i}$ factor depends only on the molar mass of $i$-mer $\left(M_{i}\right)$ in an exponential way. The selectivity of the separation is related to $\sigma$ and decreases with pressure at constant temperature. ${ }^{10,15,19}$ The constant $\beta$ is related to the ratio between the volumes of coexisting phases. ${ }^{17}$

Fractionation of PEO have been performed in supercritical $\mathrm{CO}_{2} \cdot{ }^{19}$ The highest solubilized $M_{i}$ is up to $850 \mathrm{~g} \mathrm{~mol}^{-1}$ and $\log K$ vs. $M_{i}$ is linear if $M_{i}$ exceeds $400 \mathrm{~g} \mathrm{~mol}^{-1}$. Below this critical value, the effects due the end-groups are responsible for the unexpected behavior 


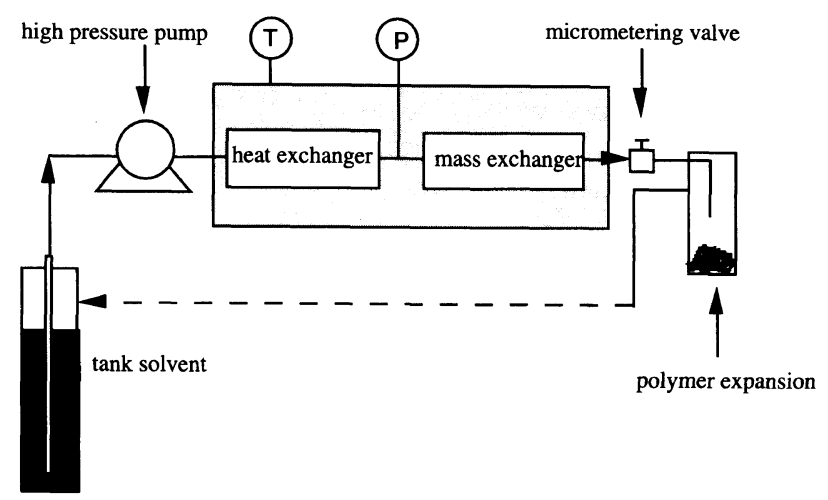

Figure 1. Schematic experimental setup used for the fractionation of poly(ethylene oxide) by supercritical R142b.

of the mass-based partition coefficient.

In this paper, we have chosen to use 1-chloro-1,1difluoroethane (R142b) as supercritical fluid for fractionation following the studies of the fractionation of polystyrene in this solvent ${ }^{15,20}$ and the state equation determination. ${ }^{21}$ In a first approach, linear PEO samples have been fractionated as a function of their molar mass. Afterwards, the separation of linear PEO contaminants (either of the same size as that of the branches of PEO stars or of the same molar mass as that exhibited by PEO stars) has been investigated with the view to obtain pure PEO star samples.

\section{EXPERIMENTAL}

All the chemical reagents used in this work were purchased from Aldrich. Synthesis and purification of different compounds were previously published. ${ }^{3}$

The apparatus which was used for SCF fractionation up to $40 \mathrm{MPa}$ and $400^{\circ} \mathrm{C}$ is presented on Figure 1 . It essentially consists of a high pressure pump (Thermoproduct Separation) and a mass exchanger. The solvent is pressurized in liquid phase up to the required pressure then it flows through a heat exchanger which brings the fluid to the required temperature. The flow rate is adjusted by a micrometering valve (Autoclave Engineers) located at the exit of the apparatus. The flow rate ranges from 2 to $10 \mathrm{ml} \mathrm{min}^{-1}$.

The mass exchanger is a column of $0.8 \mathrm{~cm}$ diameter and $20 \mathrm{~cm}$ length. It is filled with a glass packed bed of $0.2 \mathrm{~cm}$ diameter. A glass pool plug is placed at the inlet and the outlet of the column to avoid the dragging of polymer by the flowing fluid. For each experimental run, the column is charged with $1 \mathrm{~g}$ of polymer, and the solubilized fraction is collected in the dry and solid form by the expansion of the fluid.

The characterization of linear and star PEO samples was performed by a size exclusion chromatography (SEC) using a JASCO HPLC pump type 880-PU, Tosoh HAAS TSK Gel columns and a Varian RI-3 refractive index detector. Some $\bar{M}_{w}$ values were obtained from a SEC instrument equipped with a laser light scattering detector. SEC were run with either Tetrahydrofuran (THF) or water as eluent.

Freon R142b (1-chloro-1,1-difluoroethane) is provided by Dehon service ( $98 \%$ purity). Its critical temprature and critical pressure are respectively $137^{\circ} \mathrm{C}$ and $4.25 \mathrm{MPa}$.
The properties of $\mathrm{R} 142 \mathrm{~b}$ are taken from a previous paper devoted to the study of this solvent. ${ }^{21}$

\section{RESULTS AND DISCUSSION}

The first series of experiments were meant to calibrate the fractionation apparatus and to establish the plot giving the molar mass of the extracted fraction as a function of pressure. Several linear PEO samples whose molar masses ranged from 500 to $20000 \mathrm{~g} \mathrm{~mol}^{-1}$ were mixed so as to make a sample of very broad distribution of molar masses and then fractionated as a function of pressure. The experiments were performed at $150^{\circ} \mathrm{C}$ in a pressure range varying from 8 to $40 \mathrm{MPa}$ with $\mathrm{R} 142 \mathrm{~b}$ as solvent.

$\mathrm{R} 142 \mathrm{~b}$ is considered as an environmentally safe refrigerant. It exhibits a low ozone depletion potential (0.06) and a low halocarbon global warning potential $(0.41)$ as well. It is slightly inflammable, chemically inert to usual metals, plastic materials and elastomers employed in industry and it has low toxicity. It is the main intermediate compound in the synthesis of poly(vinylidene fluoride) (PVDF). It is also used as aerosol propellers of perfumes and shaving foams, as plastic foam blowing agent (polyethylene and polystyrene foams) and in industrial air conditioning. Due to its great stability in supercritical conditions, it was also considered as a potential solvent in supercritical extraction. In contrast to $\mathrm{CO}_{2}$ which is a nonsolvent of polystyrene (PS), R142b is a good solvent for this type of polymer as it can solubilize PS sample of a molar mass of $20000 \mathrm{~g} \mathrm{~mol}^{-1}$ at $150^{\circ} \mathrm{C}$ and $35 \mathrm{MPa}$.

The $M_{n}$ value corresponding to each fraction isolated was found to increase with pressure as classically observed for polymer chains in supercritical fluids. The maximum molar mass of the solubilized fraction, or "cutoff-mass" $\left(M_{\mathrm{c}}\right)$ for each pressure, is show in Figure 2. $M_{\mathrm{c}}$ corresponds to the molar mass of the slice that exhibits the lowest elution volume in the SEC eluogram.

Even though the calibration curve shown in Figure 2 gives the optimum pressure to apply for the extraction of species of given molar mass, the problem becomes more complex when the sample to fractionate contains species of different architectures. The fractionation of a binary mixture (noted $\left.\mathrm{M}_{1}\right)$ made of three-arm stars $\left(\bar{M}_{n}=\right.$ $\left.2800 \mathrm{~g} \mathrm{~mol}^{-1}\right)$ and of linear chains $\left(\bar{M}_{n}=900 \mathrm{~g} \mathrm{~mol}^{-1}\right)$ was thus attempted. In this case the contaminating chain was chosen so as to exhibit the same molar mass as that of the star branches. This situation of star contamination by linear chains is encountered whenever the plurifunctional initiator is not carefully dried and contains protonic impurities. Upon deprotonation of all protonic functions and addition of ethylene oxide, not only the branches of the star grow but also linear chains, which have to be subsequently removed. In the binary mixture $\left(\mathrm{M}_{1}\right)$, the content in linear materials was purposely raised to $50 \%$ in weight (Table I) so as to investigate the selectivity of this fractionation technique.

As evidenced in Figure 3, the separation of linear chain $\left(\bar{M}_{n}=900 \mathrm{~g} \mathrm{~mol}^{-1}\right)$ from the star structure $\left(\bar{M}_{n}=2800\right.$ $\mathrm{g} \mathrm{mol}^{-1}$ ) occurs as expected upon applying a pressure of $6 \mathrm{MPa}$ shown that linear chains of $900 \mathrm{~g} \mathrm{~mol}^{-1}$ were selectively extracted. 


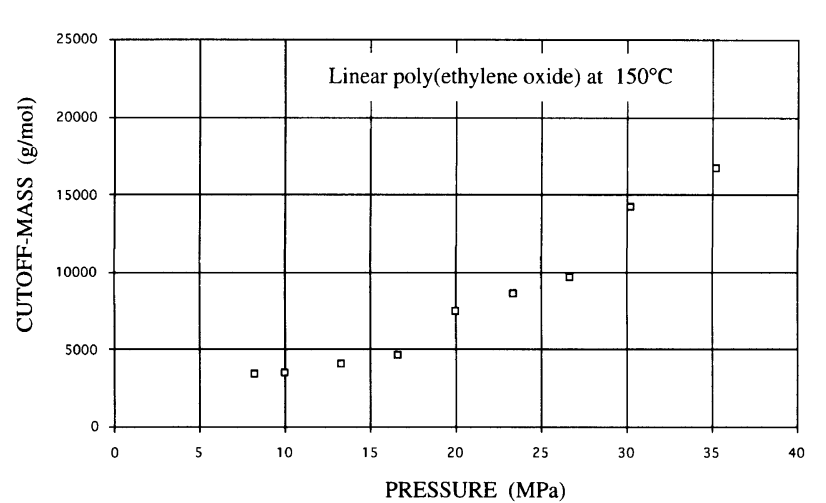

Figure 2. Cut-off-mass of linear poly(ethylene oxide) in R142b as a function of pressure at $150^{\circ} \mathrm{C}$.

Table I. Number average molar mass and composition (\% in weight) of star and linear poly(ethylene oxide) binary mixtures

\begin{tabular}{cccccc}
\hline & \multicolumn{2}{c}{$\begin{array}{c}\text { Linear } \\
\text { poly(ethylene oxide) }\end{array}$} & & \multicolumn{2}{c}{$\begin{array}{c}\text { Star } \\
\text { poly(ethylene oxide) }\end{array}$} \\
\cline { 2 - 3 } \cline { 5 - 6 } & $\bar{M}_{n}$ & $\mathrm{wt} \%$ & & $\bar{M}_{n}$ & $\mathrm{wt} \%$ \\
\hline $\mathrm{M}_{1}$ & 900 & 50 & & 2800 & 50 \\
$\mathrm{M}_{2}$ & 10000 & 20 & & 10330 & 80 \\
\hline
\end{tabular}

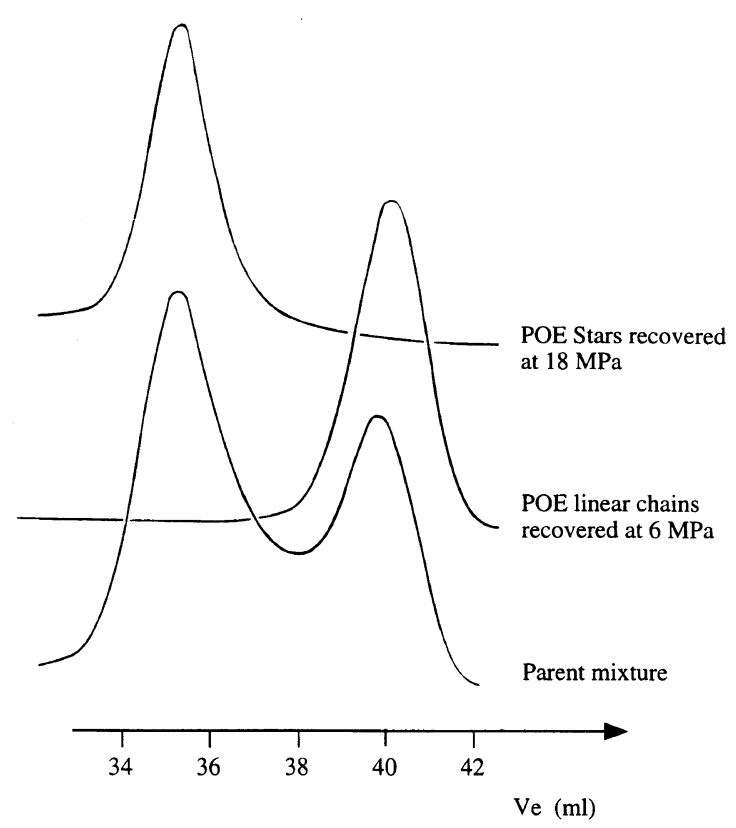

Figure 3. Fractionation of binary mixture $M_{1}(50 / 50 \%$ in mass $)$ of poly(ethylene oxide) stars $\left(\bar{M}_{n}=2800 \mathrm{~g} \mathrm{~mol}^{-1}\right)$ and linear chains of poly(ethylene oxide) $\left(\bar{M}_{n}=900 \mathrm{~g} \mathrm{~mol}^{-1}\right)$ as a function of pressure at $150^{\circ} \mathrm{C}$ and $5 \mathrm{ml} \mathrm{min}^{-1}$.

Another case involving star molecules and linear chains was also investigated. A binary mixture $\left(\mathrm{M}_{2}\right)$ containing PEO stars and linear of about the same molar mass was then investigated (Table I). The fractionation using $\mathrm{R} 142 \mathrm{~b}$ as supercritical fluid was effected at $25 \mathrm{MPa}$. The value of the pressure to apply was drawn from the calibration curve (Figure 2) established for linear chains. This curve shows that for the selective extraction of materials exhibiting mass lower $10000 \mathrm{~g} \mathrm{~mol}^{-1}$, pressure of $25 \mathrm{MPa}$ is required. The studies have been conducted
Table II. Recovered poly(ethylene oxide) star as a function of time at $25 \mathrm{MPa}, 150^{\circ} \mathrm{C}, 6 \mathrm{ml} \mathrm{min}{ }^{-1}$, and for an initial load of $1 \mathrm{~g}$ of $\mathrm{M}_{2}$ binary mixture

\begin{tabular}{cc}
\hline Time/min & $\begin{array}{c}\text { Recovered } \\
\text { poly(ethylene oxide }) / g\end{array}$ \\
\hline 0 & 0 \\
20 & 0.39 \\
40 & 0.56 \\
60 & 0.68 \\
80 & 0.77 \\
\hline
\end{tabular}

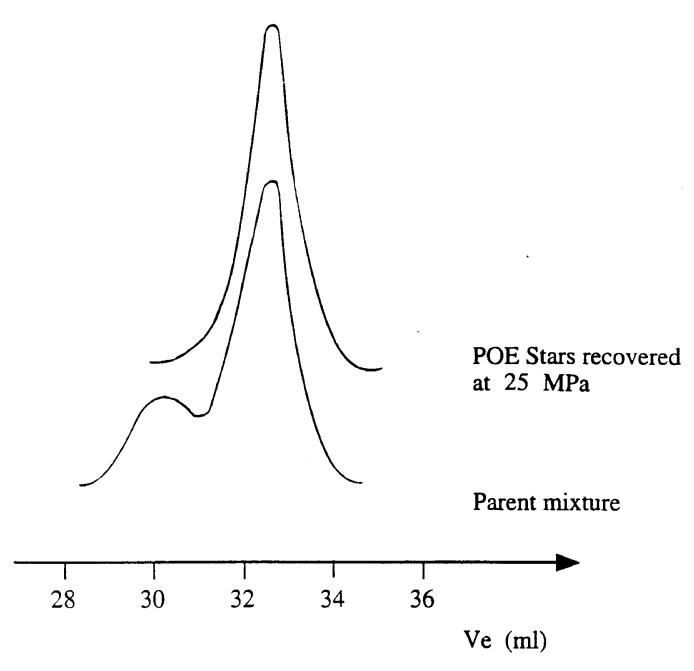

Figure 4. Fractionation of a binary mixture $M_{2}(80 / 20 \%$ in mass) of poly(ethylene oxide) stars $\left(\bar{M}_{n}=10330 \mathrm{~g} \mathrm{~mol}^{-1}\right)$ and linear chains of poly(ethylene oxide) $\left.\left(\bar{M}_{n}=10000 \mathrm{~g} \mathrm{~mol}^{-1}\right)\right)$ at $25 \mathrm{MPa}, 150^{\circ} \mathrm{C}$, and $6 \mathrm{ml} \mathrm{min}^{-1}$.

with an initial load of $1 \mathrm{~g}$ of binary mixture. Table II shows the increase of the amount of PEO stars recovered $\left(\mathrm{M}_{2}\right)$ as a function of time, for a pressure of $25 \mathrm{MPa}$ and a flow rate of $6 \mathrm{ml} \mathrm{min}{ }^{-1}$. The separation of linear contaminants from star polymers was actually performed with an efficiency higher than $90 \%$ in weight. Figure 4 exhibits the SEC eluograms of recovered polymers compared with that of the parent mixture. These results call for two comments.

The first one concerns the mass transfer and the fact that the fractionation efficiency is very high. Indeed, the kinematic viscosity $(\nu=\eta / \rho ; \eta$ and $\rho$ are viscosity and density of fluid respectively) is very low in the supercritical region. This point is worth noting for masstransfer applications, since natural convection effects are inversely proportional to the square of the kinematic viscosity. ${ }^{22}$ However, the fractionation of a $i$ mer becomes more difficult when this $i$-mer concentration in the polymer rich phase decreases as shown by eq 1 . This phenomena is classically observed in liquid extraction. Equation 1 shows that $K_{i}$ is constant and is equal to $w_{i}^{\prime} / w_{i}$ which indicates that the transfer in the fluid phase decreases with $w_{i}$. As a result, the last residual amounts of impurities are difficult to extract. From a general point of view, the best process for material purification consists in extracting by supercritical fluids the major part of the product of intereset so as to obtain it as pure as possible, the species remaining in the stationary phase being essentialy impurities. This was 
indeed performed with M2 mixture.

The second comment is related to the thermodynamic properties of polymers which can be very efficiently fractionated as a function of their molar size using superctitical fluids, regardless of their molar mass and/or their overall architecture. The partition process cannot be interpreted as being due to steric exclusion but rather to a solubility process that depends of the fluid density and the hydrodynamic volume of the polymer at a given temperature. The large compressibility of superctitical fluids influences the rates of solubility with respect to temperature and pressure. ${ }^{23}$ This behavior is classically observed for polymeric materials and it is known that the $i$-mer solubility increases with fluid density at constant temperature. ${ }^{15}$ When applied to polymers in supercritical fluid models such as the statistical associating fluid theory (SAFT) ${ }^{24}$ or the Sanchez-Lacombe theory ${ }^{25}$ indeed predict such behavior. These models show that the demixing pressures on polymers in supercritical fluids increases with the molar mass of the sample. The Sanchez-Lacombe model defines the intermolecular and intramolecular interactions and the close-packed volume of a $i$-mer. The intramolecular interactions for the star structure are expected to be higher than for the linear structure of same molar mass giving rise to a low hydrodynamic volume on the star structure than that of the linear chain. As evidenced by steric exclusion chromatography, star structures exhibiting the same molar mass as that of a linear equivalent is eluted at higher elution times. Because of its lower hydrodynamic volume the star structure exhibits a lower apparent mass. Therefore this star macromolecule will be solubilized at a lower pressure than would be its homologous linear structure. The point that really matters for separation by supercritical fluids is the hydrodynamic volume of the different species to fractionate. In other words, two polymers of the same chemical nature and molar masses but different topologies can be separated off, provided their respective molecular dimensions strongly differ. The driving force for an effective separation by supercritical fluids depends on the temperature and density of the solvent, and surprisingly on the hydrodynamic volume of the polymer rather than its molar mass.

This new technique of fractionation that was shown to function for the separation of two different architectures can be used as a preparative method. Indeed, the purification of $1 \mathrm{~g}$ of star/linear mixture with $\bar{M}_{n}=$ $2800 \mathrm{~g} \mathrm{~mol}^{-1}$, was completed in $80 \mathrm{~min}$ time with a flow rate of fluid of $10 \mathrm{ml} \mathrm{min}^{-1}$. The amount of purified PEO star recovered in the mass exchanger is above $90 \%$.
These results are remarkable when compared to other techniques of fractionation (dialyze or SEC) which either take much more time or only afford very small amounts of fractionated polymers.

\section{REFERENCES}

1. Y. Gnanou, J. Macromol. Sci., Rev. Macromol. Chem. Phys., C36(1), 77 (1996).

2. E. Cloutet, J. L. Fillant, D. Astruc, and Y. Gnanou, "Macromolecular Engineering: Contemporary Themes," M. Mishara, Ed. Plenum Press, New York, N.Y., 1995, p 47.

3. Y. Gnanou, P. Lutz, and P. Rempp, Makromol. Chem., 189, 2885 (1988).

4. J. L. Six and Y. Gnanou, Macomol. Symp., 95, 137 (1995).

5. D. Yen, S. Raghavan, and E. Merrill, Macromolecules, 29, 8977 (1996).

6. F. Cansell and J. P. Petitet Ed., "Fluides Supercritiques et Matériaux," Lavoisier Publishing, Paris, 1995.

7. I. Yilgor, J. E. McGrath, and V. J. Krukonis, Polym. Bull., 12, 491 (1984).

8. I. Yilgor, J. E. McGraph, and V. J. Krukonis, Polym. Bull., 12, 499 (1984).

9. V. J. Krukonis, Polym. News, 11, 7 (1985).

10. S. K. Kumar, S. P. Chhabria, R. C. Reid, and U. W. Suter, Macromolecules, 20, 2550 (1987).

11. K. M. Scholsky, V. M. o'Connor, and C. S. Weiss, J. Appl. Polym. Sci., 33, 2925 (1987).

12. E. P. Schmitz and E. J. Klesper, J. Supercrit. Fluids, 3, 29 (1990).

13. J. J. Watkins, V. J. Krukonis, P. D. Condo, D. Pradham, and P. J. Ehrlich, J. Supercrit. Fluids, 4, 24 (1991).

14. E. Kiran and W. Zhuang, Polymer, 33, 5259 (1992).

15. Ph. Desmarest, M. Hamedi, R. Tufeu, and F. Cansell, Third Inter. Symp. on Supercritical Fluids, I.S.A.S.F., Nancy, France, 3, 287 (1994).

16. M. Radosz, M. Banaszak, C. K. Chen, and C. J. Gregg, Third Inter. Symp. on Supercritical Fluids, I.S.A.S.F., Nancy, France, 3, 279 (1994)

17. P. J. Flory, "Principles of Polymer Chemistry," Cornell University Press, Ithaca, N.Y., 1953, p. 559

18. R. Koningsveld, W. H. Stockmayer, J. W. Kennedy, and L. A. Kleintjens, Macromolecules, 7, 73 (1974).

19. M. Daneshwar and E. Gulari, J. Supercrit. Fluids, 5, 143 (1992).

20. M. Hamedi, F. Cansell, Desmarest, and R. Tufeu, Third Inter. Symp. on Supercritical Fluids, I.S.A.S.F., Nancy, France, 1, 285 (1994).

21. Ph. Botella, J. L. Six, Y. Gnanou, B. Le Neindre, Y. Garrabos, R. Tufeu, and F. Cansell, High Temperatures and High Pressures, 29 (1997), in press.

22. P. G. Debenedetti and R. C. Reid, Am. Inst. Chem. Eng. J., 32, 2034 (1986)

23. C. A. Ekert, D. H. Ziger, K. P. Johnson, and S. Kim, J. Phys. Chem., 90, 2738 (1986).

24. C. K. Chen, M. A. Marco, and M. Radosz, Ind. Eng. Chem. Res., 32, 3123 (1993).

25. E. Kiran, Y. Xiong, and W. Zhuang, J. Supercritical Fluids, 6, 193 (1993). 